Trauma Berufskrankh 2008 · 10[Suppl 1]:75-77 DOI 10.1007/s10039-007-1264-x

Online publiziert: 30 . August 2007

(c) Springer Medizin Verlag 2007
A. Nienhaus ${ }^{1} \cdot$ K. Kromark ${ }^{1} \cdot$ M. Raulf-Heimsoth ${ }^{2} \cdot$ V. van Kampen ${ }^{2} \cdot$ R. Merget $^{2}$

${ }^{1}$ Abteilung Grundlagen der Prävention und Rehabilitation, Berufsgenossenschaft für Gesundheitsdienst und Wohlfahrtspflege, Hamburg

${ }^{2}$ Forschungsinstitut für Arbeitsmedizin der Deutschen Gesetzlichen Unfallversicherung (BGFA), Institut der Ruhr-Universität, Bochum

\section{Berufsbedingte Latexallergie}

\section{Nachhaltige Entwicklungen in Berufs- und Privatleben}

\section{Hintergrund und Fragestellung}

Für Beschäftigte in medizinischen Bereich besteht aufgrund des häufigen und lang andauernden Kontakts zu Gegenständen aus Naturlatex ein erhöhtes Risiko, an einer Latexallergie zu erkranken. Studien beschrieben eine Prävalenz der Sensibilisierung gegen Naturlatex im medizinischen Berufen von bis $\mathrm{zu} 17 \%$ [7]. Hauptallergenträger sind gepuderte Latexhandschuhe $[2,6]$. Die Zahl der bestätigten BK 5101-Fälle stieg von etwa 300 im Jahr 1995 auf etwa 900 im Jahr 1998. Seitdem ist der Trend rückläufig, was vermutlich auf erfolgreiche Präventionsmaßnahmen zurückzuführen ist $[1,3,4]$. Darüber hinaus wurde in der Literatur der Rückgang der Sensibilisierung gegenüber naturlatexhaltigen Produkten nach der Implementierung von Präventionsmaßnahmen beschrieben [5].

Ziel der vorliegenden Studie war es, Gesundheit, Lebensqualität und Aspekte der Berufstätigkeit bei Versicherten mit anerkannter Latexallergie zu beschreiben. Die Untersuchung der Krankheitsverläufe soll einen Überblick über die Nachhaltigkeit von Präventions- und Rehabilitationsmaßnahmen geben.

\section{Studiendesign und Untersuchungsmethoden}

Es wurden 329 Versicherte mit einer bestätigten Latexallergie (BK 4301 und BK
Abb. 2 Latexallergie, Einfluss auf die Lebensqualität; Hautallergie BK 5101: $n=147$; Atemwegsallergie BK 4301: $n=98$
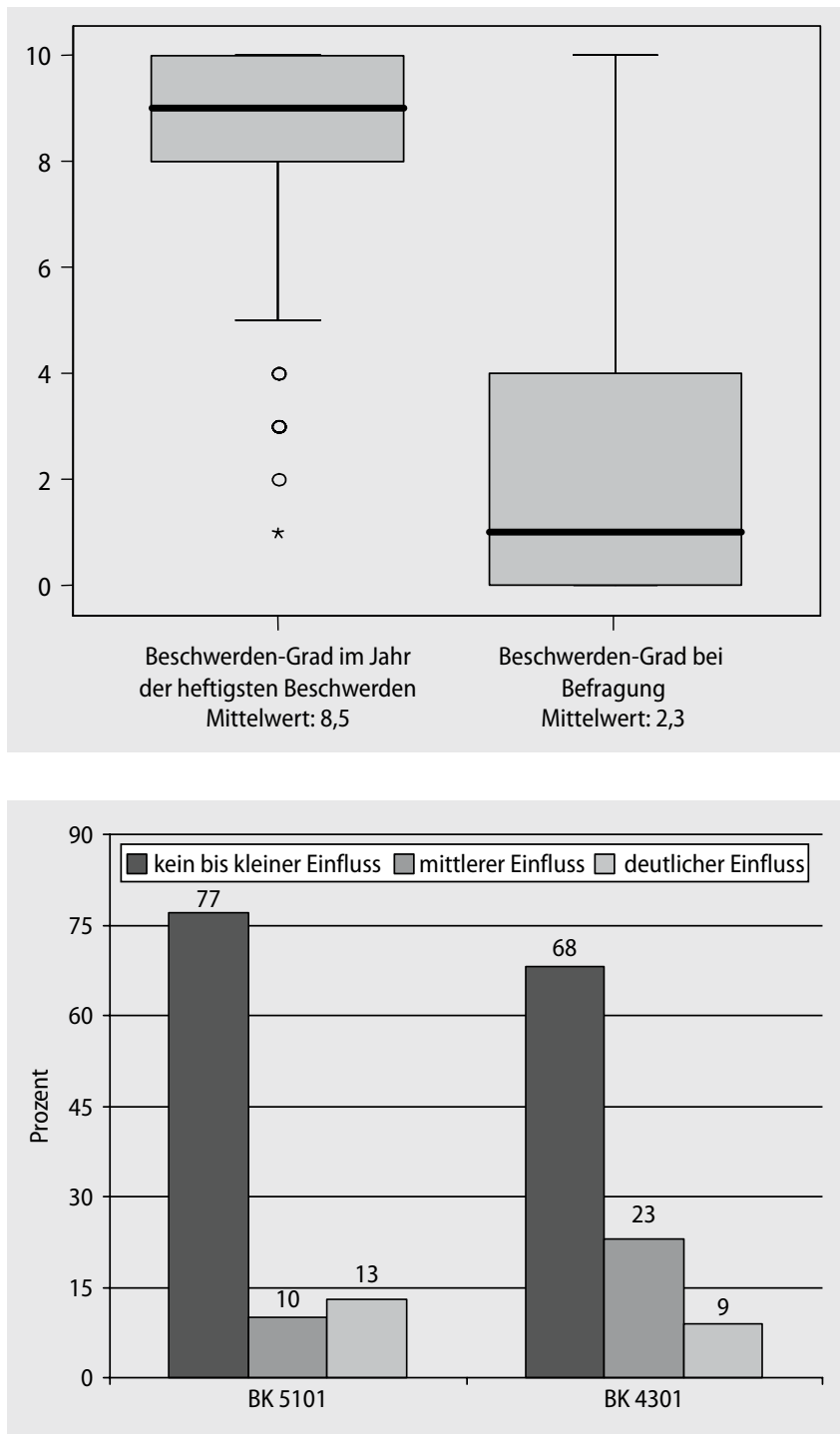
Trauma Berufskrankh 2008 · 10[Suppl 1]:75-77 DOI 10.1007/s10039-007-1264-x

(c) Springer Medizin Verlag 2007

\section{A. Nienhaus · K. Kromark · M. Raulf-Heimsoth ·V. van Kampen · R. Merget Berufsbedingte Latexallergie. Nachhaltige Entwicklungen in Berufs- und Privatleben}

\section{Zusammenfassung}

Die Vermeidung gepuderter Latexhandschuhe gilt als wichtige Maßnahme zur Prävention von latexbedingten Haut- und Atemwegsallergien bei Beschäftigten im Gesundheitsdienst. Ob sie auch für Beschäftigte mit einer bereits bestehenden Allergie ausreichend ist, wurde überprüft. Mit einem standardisierten Fragebogen wurden 329 Beschäftigte im Gesundheitswesen mit einer beruflich bedingten Latexallergie zur Lebensqualität, zur Allergenkarenz und zu allergiebezogenen Beschwerden befragt. Letztere waren zum Zeitpunkt der Befragung deutlich rückläufig. Gepuderte Latexhandschuhe wurden von allen Befragten gemieden. Keine allergiebezogenen Symptome hatten 77\% der Patienten mit einer Hautallergie und 68\% derjenigen mit einer Atemwegsallergie. Die Vermeidung von gepuderten Latexhandschuhen ist daher auch bei Versicherten mit einer bestehenden Latexallergie eine effektive Präventionsmaßnahme.

\section{Schlüsselwörter}

Gepuderte Latexhandschuhe - Latexbedingte Hautreaktionen - Latexbedingte Atemwegsallergien · Beschäftigte im Gesundheitsdienst · Prävention

\section{Occupationally induced latex allergy. Enduring developments in professional and private life}

\begin{abstract}
Avoiding powdered latex gloves is generally accepted as being an important way for health service employees to avoid latexlinked allergies affecting the skin and respiratory tract. A questionnaire study was performed to test whether this preventive measure would also be adequate to prevent symptoms in employees with an existing allergy. Using a standardised questionnaire, 329 health service employees with occupational latex allergy were asked about their quality of life, periods without contact with allergens and symptoms related to allergens. At the time when the questionnaire was ad-
\end{abstract}

ministered the symptoms were declining markedly. Powdered latex gloves were avoided by all subjects. Seventy-seven per cent of subjects with skin allergy and $68 \%$ of subjects with respiratory tract allergy had no allergic symptoms. We conclude therefore that avoiding latex gloves is also an effective preventive measure for insured subjects with existing latex allergy.

\section{Keywords}

Powdered latex gloves - Latex-induced cutaneous reactions - Latex-induced airway reactions · Healthcare workers · Prevention
5101) der Meldejahre 1996-2004 per Zufallsauswahl angeschrieben. Die Rücklaufquote betrug 60\%. Von diesen 196 Personen wurden 14 aufgrund einer nicht gesicherten Diagnose ausgeschlossen. Der Anteil der Pflegekräfte unter den verbleibenden 182 Versicherten betrug im Jahr der BK-Meldung 59\%.

Die mittlere Zeitspanne zwischen dem Jahr der BK-Meldung und der Befragung betrug 6,8 Jahre. Die Versicherten wurden mittels eines standardisierten, selbst auszufüllenden Erhebungsinstruments befragt. Der Fragebogen gliederte sich in 4 Teilbereiche:

a) Fragen zur Person, zur beruflichen Tätigkeit und zum Allergiestatus,

b) Informationen zu privaten und beruflichen Präventionsmaßnahmen,

c) Fragen zur Lebensqualität und

d) Fragen zum Aspekt „Arbeit und Gesundheit".

Im Teilbereich a gaben die Versicherten das Ausmaß ihrer Latexallergiebeschwerden auf einer Skala von o-10 an. In den Teilbereichen b und ckamen validierte Erhebungsinstrumente zum Einsatz. Dabei handelt es sich um den Dermatology Life Quality Index (DLQI), den Mini Asthma Quality of Life Questionnaire (MiniAQLQ) und den Work Ability Index (WAI). Die beiden Instrumente zur Erfassung der Lebensqualität beziehen sich auf den 2-wöchigen Zeitraum vor der Befragung und wurden ausschließlich von Personen ausgefüllt, die in den 6 Monaten vor der Befragung Haut- bzw. asthmatische Beschwerden aufwiesen.

\section{Ergebnisse}

Mehr als 2/3 (65\%) der Versicherten übten bei der Befragung dieselbe oder eine vergleichbare Tätigkeit aus wie im Meldejahr, eine andere Tätigkeit wurde von $10 \%$ ausgeübt. 1/4 der Versicherten (24\%) waren bei der Befragung nicht mehr erwerbstätig. Von diesen 44 Personen gaben 18 ihre Erwerbstätigkeit aufgrund der Latexallergie auf.

Der Mittelwert der latexallergiebedingten Beschwerden lag zum Zeitpunkt der stärksten Beschwerden auf einer Skala von $0-10$ bei 8,5 und bei der Befragung bei 2,3 (- Abb. 1). In $53 \%$ der Fälle war 
es zu einer Senkung des Beschwerdegrads um 7-10 Punkte gekommen. Lediglich bei $6 \%$ der Befragten waren eine Verschlechterung bzw. Stagnierung des Beschwerdegrads zu verzeichnen.

Gepuderte Latexhandschuhe wurden von allen Befragten gemieden. Eine gute Allergenkarenz gelang bei $84 \%$ der Befragten im privaten Bereich und bei $61 \%$ im beruflichen Bereich. Bei $13 \%$ der Versicherten mit einer Hautallergie (BK 5101) führte die Latexallergie zu einer deutlichen Einschränkung der Lebensqualität. Die Lebensqualität durch latexallergiebedingtes Asthma (BK 4301) war bei 9\% der Versicherten mit einer BK 4301 deutlich eingeschränkt (• Abb. 2).

\section{Diskussion}

Der deutliche Rückgang der Prävalenz berufsbedingter Latexallergien wird in der Literatur u. a. auf Allergenkarenz zurückgeführt. Diese Einschätzung wird trotz der subjektiven Symptomerfassung durch die vorliegende Studie bestätigt. Allerdings geben einige Beschäftigte weiterhin latexbezogene Symptome an. Hier ist eine genauere labortechnische Abklärung mit den fortentwickelten diagnostischen Möglichkeiten notwendig.

\section{Schlussfolgerungen}

Puderfreie Handschuhe sind hinsichtlich der Lebensqualität beim größten Teil der Versicherten mit Latexallergie eine ausreichende Präventionsmaßnahme.

\section{Korrespondenzadresse}

\section{PD Dr. A. Nienhaus}

Abteilung Grundlagen der Prävention und Rehabilitation, Berufsgenossenschaft für Gesundheitsdienst und Wohlfahrtspflege, Pappelallee 35-37, 22089 Hamburg

albert.nienhaus@bgw-online.de

Interessenkonflikt. Der korrespondierende Autor gibt an, dass kein Interessenkonflikt besteht.

\section{Literatur}

1. Allmers H, Schmengler J, Skudlik C (2002) Primary prevention of natural rubber latex allergy in the German health care system through education and intervention. J Allergy Clin Immunol 110: 318323
2. Baur X, Chen Z, Raulf-Heimsoth M et al. (1988) Protein und allergen content of various natural latex articles. Allergy 52: 661-664

3. Latza U, Haamann F, Baur X (2005) Effectiveness of a nationwide interdisciplinary preventive programme for latex allergy. Int Arch Occup Environ Health 78: 394-340

4. Raulf-Heimsoth M, Sander I, Rihs HP et al. (2003) Latex allergy: state of the art. Aktuelle Dermatol 29: 176-184

5. Ruëff F, Schöpf P, Putz K et al. (2004) Effect of reduced exposure on natural rubber latex sensitization in health care workers. Ann Allergy Asthma Immunol 92: 530-537

6. Williams P, Buhr M, Weber R et al. (1995) Latex allergen in respirable particulate air pollution. J Allergy Clin Immunol 95: 88-95

7. Yassin MS, Lierl MB, Fisher TJ et al. (1994) Latex allergy in hospital employees. Ann Allergy 72: 245249 